ПРИМЕНЕНИЕ МЕТОДА ВОССТАНОВЛЕНИЯ

ТЕМПЕРАТУРНО-ВЫСОТНОГО ПРОФИЛЯ

СОЛНЕЧНОЙ АТМОСФЕРЫ К МНОГОЧАСТОТНЫМ

РАДИОНАБЛЮДЕНИЯМ НЕКОТОРЫХ АКТИВНЫХ ОБЛАСТЕЙ

\author{
Макоев Г.А. ${ }^{1,2}$, Ступишин А. Г. ${ }^{2}$, Кальтман Т.И. ${ }^{1}$ \\ ${ }^{1}$ Санкт-Петербургский филиал САО РАН, Санкт-Петербург, Россия \\ ${ }^{2}$ Санкт-Петербургский государственный университет, Санкт-Петербург, Россия
}

\title{
APPLICATION OF THE METHOD OF OBTAINING THE HEIGHT-TEMPERATURE PROFILE OF THE SOLAR ATMOSPHERE TO MULTI-FREQUENCY RADIO OBSERVATIONS OF SOME ACTIVE REGIONS
}

\author{
Makoev G.A. ${ }^{1,2}$, Stupishin A.G. ${ }^{2}$, Kaltman T.I. ${ }^{1}$ \\ ${ }^{1}$ St. Petersburg filiation of SAO RAS, St.-Petersburg, Russia \\ ${ }^{2}$ St. Petersburg State University, St.-Petersburg, Russia
}

\begin{abstract}
This report demonstrates the application of the method of obtaining the heighttemperature profile over the active regions to the microwave range RATAN-600 data in for some of them. The iterative method and some of its generalizations are described, which make it possible to consider the inhomogeneities in the parameters of the solar atmosphere over the selected areas of the active region (umbra and penumbra) in the distribution of polarized radio emission. These generalizations are consistently applied to the observational data obtained on the RADIO telescope RATAN-600 for the active regions. For each active region the estimation of bremsstrahlung emission was included into model and that estimation led us to more correct height-temperature profiles. For each of these regions, realistic estimates of the temperature in the lower corona (lower temperatures above the shadow and higher above the penumbra of the active region) were obtained, which are well consistent with modern ideas about the solar atmosphere.
\end{abstract}

DOI: 10.31725/0552-5829-2021-189-192

\section{1. Введение}

В работе анализируются результаты применения метода реконструкции параметров солнечной атмосферы над активными областями (АО). Метод основан на моделировании циклотронного излучения по данным многочастотных наблюдений радиотелескопа РАТАН-600 и реконструкции магнитного поля из наблюдений SDO/HМI. Такой метод был предложен в [1] и далее развит в [2,3]. Метод позволяет восстановить высотный профиль электронной температуры (и, до некоторой степени, электронной плотности) в сильных магнитных полях над пятнами. В данной статье метод дополнен учетом тормозного излучения во флоккульных областях (без циклотронного излучения) с использованием модели [4]. Показано применение метода на примере нескольких активных областей. 


\section{2. Применение метода к АО 12470}

Рассмотрим сначала более подробно применение метода к наблюдательным данным РАТАН-600 для области 12470 (за 2015/12/18), состоящей из большого одиночного пятна и окружающей его флоккульной площадки.

\section{1. Использование маски для разделения источника на компоненты}

Метод, описанный в [2], мы дополнили применением маски (рис. 1), полученной на основе измерений магнитного поля SDO/HMI и излучения в континууме (SDO/Continuum_NoLimbDark). Маска дает возможность восстанавливать профили температуры и электронной плотности раздельно для тени и для полутени, а также учитывать тормозное излучение флоккульной компоненты, задавая для нее высотные профили температуры и плотности согласно модели [4].
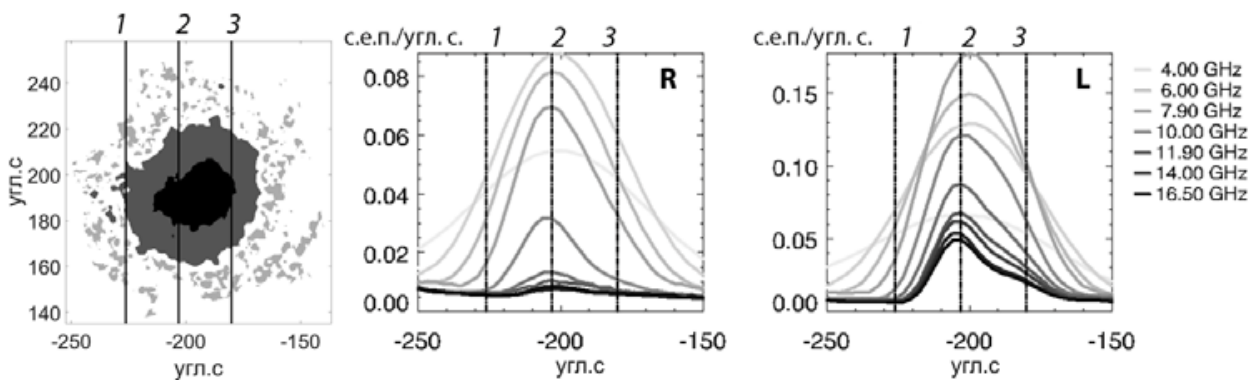

Рис. 1. Слева: маска для разделения на тень (черная), полутень (серая) и флоккульную компоненту (светло-серая) АО 12470, вертикальные линии 1-3 соответствуют выбранным на сканах (справа) точкам. В центре и справа - сканы АО 12470, полученные 2015/12/18 на РАТАН-600 (параметры Стокса R и L).

\section{2. Учет тормозного излучения при восстановлении профиля}

Для восстановления температурно-высотного профиля были взяты наблюдательные спектры (рис. 2, слева), полученные для линий 1-3. Модельные спектры тормозного излучения (рис. 2, справа), рассчитанные

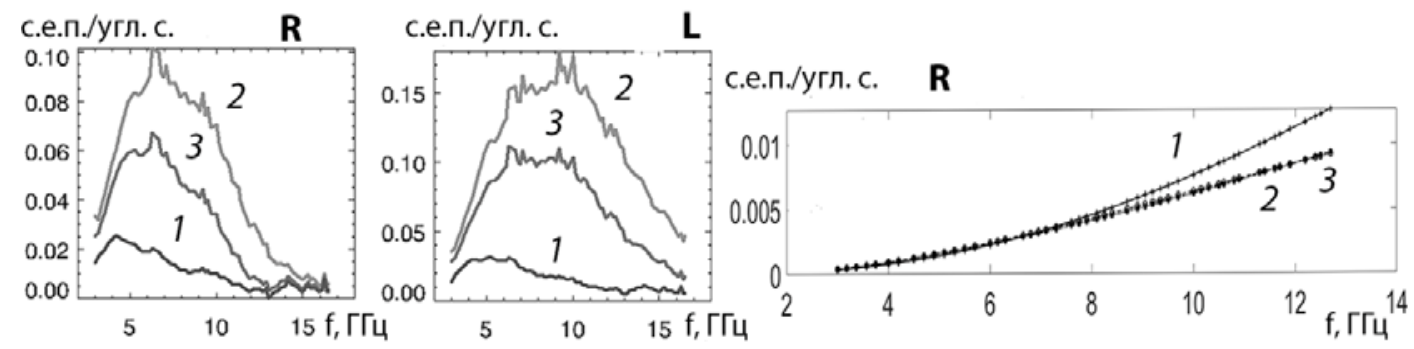

Рис. 2. Слева: спектры AO 12470 (РАTAH-600), параметры Стокса R и L), в точках 1-3 (см. маску на рис. 1). Справа: расчет тормозного излучения флоккульной компоненты.

вдоль линий 2 и 3 , практически одинаковы и расходятся с 1 на высоких частотах, так как площадь флоккульной компоненты вдоль линии 1 и, соответственно, вклад тормозного излучения, больше. Сравнение полученных результатов на рис. 3 показывает, что учет тормозного излучения позволяет получить более монотонные и реалистичные температурные профили (особенно заметно отличие в переходной зоне (ПЗ)), бо́льшую высоту ПЗ и более высокую корональную температуру для полутени, чем для тени. 


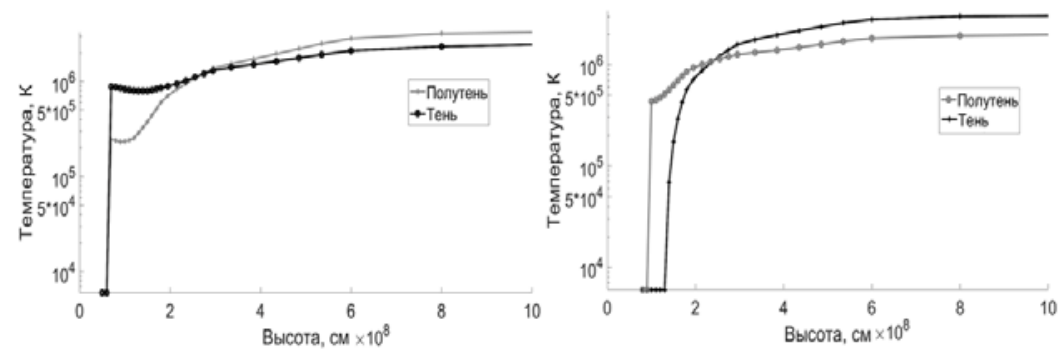

Pис. 3. Полученный температурно-высотный профиль для AR12470, слева: без учета тормозного излучения; справа: с учетом тормозного излучения.

\section{3. Применение метода к АО 11312, 12419 и 12794}

Обновленный метод восстановления температурно-высотного профиля с применением маски и с учетом тормозного излучения был также применен к трем AO (рис. 4, таблица), также на основе многочастотных спектрально-поляризационных данных РАТАН-600. AR11312 - «эталонная» область с симметричным одиночным пятном (cp. с [1]). Для нее получены гладкие профили тени и полутени с быстро растущей в ПЗ температурой. AR12419 - слабая биполярная область, интересна тем, что вблизи сформировался джет; так как ее циклотронное излучение относительно невелико, а флоккульная компонента, наоборот, развита, это затрудняет применение метода, поэтому профиль удалось восстановить только в верхней части ПЗ. Для одиночного пятна АО12794 профиль ПЗ восстановлен успешно.

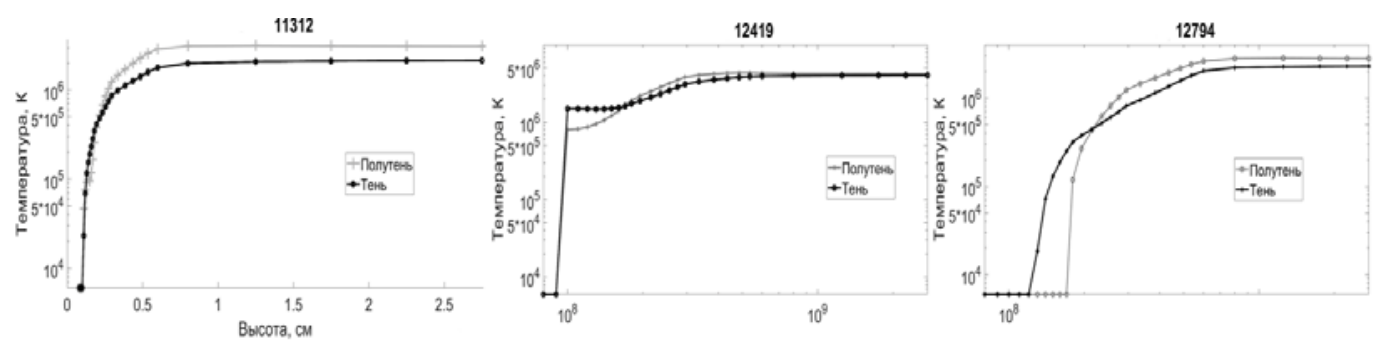

Рис. 4. Применение алгоритма к данным для активных областей с учетом тормозного излучения: a) AR11312, б) AR12419, в) AR12794

\section{4. Результаты}

Как видно из рис. 3-4, для всех представленных активных областей получено, что ПЗ полутени расположена выше ПЗ тени, и корональная температура над областью полутени выше, чем над областью тени. Полученные параметры для нижней короны показаны в табл. 1. Более достоверными являются значения температуры, а оценка плотности получена при варьировании давления $\mathrm{P}=\mathrm{NT}$ в основании короны (выбрано значение, соответствующее минимальной невязке при расчетах профиля). Полученные профили в общих чертах согласуются с моделями из работы [5]. Есть расхождения в высоте нижней переходной зоны, что может быть объяснено индивидуальными особенностями АО, но также требуется доработка метода для более уверенного определения этого сложного параметра и дальнейшее статистическое исследование. 
Таблица. Параметры АО и восстановленных профилей (NT - произведение корональной температуры и электронной плотности, $T u$ - температура «тени», $T p$ - температура «полутени», $\mathrm{Nu}$ - плотность «тени», $N p$ плотность «полутени»).

\begin{tabular}{|c|c|c|c|c|c|c|c|c|}
\hline AO & Дата & $\begin{array}{c}\text { Hale/ } \\
\text { McIntosh } \\
\text { класc }\end{array}$ & $\begin{array}{l}\text { Пло- } \\
\text { щадь, } \\
\text { м.д.п. }\end{array}$ & $N T$ & $T u, \mathbf{K}$ & $T p, \mathbf{K}$ & $\mathrm{Nu}, \mathrm{CM}^{-3}$ & $\begin{array}{c}N p \\
\mathrm{CM}^{-3}\end{array}$ \\
\hline 12470 & 015. & $\beta /$ Eko & 610 & $1 \cdot 10^{16}$ & $1.9 \cdot 10^{6}$ & $3.0 \cdot 10^{6}$ & $5.3 \cdot 10^{9}$ & $3.3 \cdot 10^{9}$ \\
\hline 11312 & 2011.10 .10 & $\alpha / H s x$ & 210 & $1 \cdot 10^{16}$ & $2.0 \cdot 10^{6}$ & $3.1 \cdot 10^{6}$ & 5.0 & $3.2 \cdot 10^{9}$ \\
\hline 12419 & 2015.09 .18 & $\beta / \mathrm{CaO}$ & 100 & $2 \cdot 10^{16}$ & $3.9 \cdot 10^{6}$ & $4.3 \cdot 10^{6}$ & $5.2 \cdot 10^{9}$ & $4.6 \cdot 10^{9}$ \\
\hline 12794 & 2020.12 .28 & $\alpha / \mathrm{Hsx}$ & 210 & $1 \cdot 10^{16}$ & $2.2 \cdot 10^{6}$ & $2.8 \cdot 10^{6}$ & $4.5 \cdot 10^{9}$ & $3.6 \cdot 10^{9}$ \\
\hline
\end{tabular}
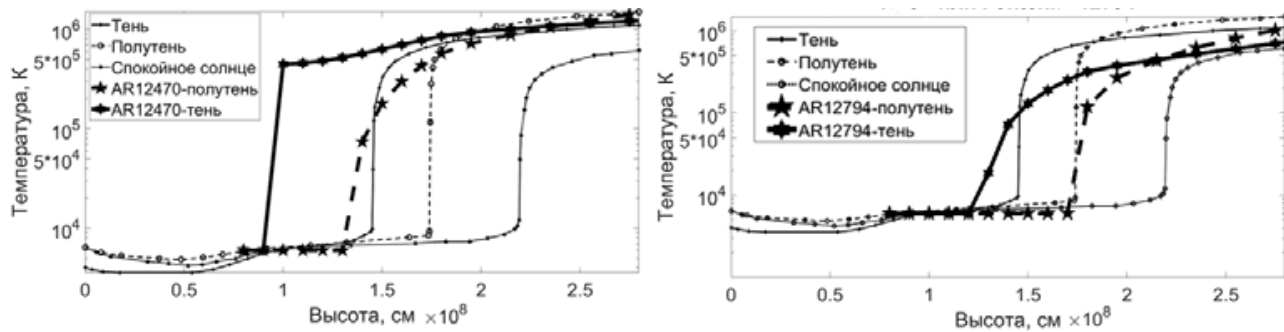

Рис. 5 Сравнение полученных профилей для тени и полутени для АО 12470 (слева) и 12794 (справа) с моделями из работы [5].

\section{5. Выводы}

Применение метода восстановления высотных профилей температуры и электронной плотности к нескольким АО, в том числе биполярным, показало достаточно хорошие результаты. Метод дополнен учетом тормозного излучения, что позволяет получить более реалистичные температурные профили. Полученные оценки (температура $1.9-3.910^{6} \mathrm{~K}$ над тенью пятна и $2.8-4.310^{6} \mathrm{~K}$ над полутенью, электронная концентрация $3.2-5.310^{9} \mathrm{~cm}^{-3}$ ) согласуются с современными представлениями о структуре и параметрах солнечной атмосферы.

Тем не менее, имеется некоторое расхождение реальных и модельных спектров, которое, по-видимому, связано с более сложной, несимметричной и многокомпонентной структурой $\mathrm{AO}$, которую только приближенно можно разделить на условно-усредненные тень, полутень и флоккульную площадку. В перспективе метод может быть применен для 2-мерных карт поляризованного радиоизлучения, полученных на инструментах с высоким спектральным и пространственным разрешением.

Работа А.С. и Т.К. поддержана грантом РФФИ 18-29-21016.

\section{Литература}

1. Stupishin A.G. et al. // Solar Phys., 2018, 293, 13.

2. Ступишин А.Г., Кальтман Т.И. // Труды конференции "Солнечная и солнечно-земная физика- 2020", СПб, 2020, с 281-284

3. Kaltman, T.I. and Stupishin, A.G. // Proc. of conf. "Ground-Based Astronomy in Russia.

21 st Century", Nizhny Arkhyz, Russia, 2020, V.3, p.409.

4. Fontenla, J., et al. // Astrophys. J., 2009, 707:482

5. Fontenla, J. et al. // Astrophys. J., 1999, V. 518, I. 1, pp. 480-499. 